\title{
Retour subreptice de l'Histoire chez quelques romanciers belges francophones (Baillon, Simenon, Savitzkaya, Toussaint)
}

\section{Retourn of History in a few Belgian francophone novelists (Baillon, Simenon, Saviztkaya, Toussaint)}

\author{
Laurent Demoulin \\ Université de Liège \\ e-mail: ldemoulin@ulg.ac.be
}

\begin{abstract}
André Baillon, Georges Simenon, Eugene Savitzkaya and Jean-Philippe Toussaint: four Belgian novelists, while portraying precisely their time, ensure generally avoid the great History. They attach indeed to depict the everyday world of men, women and children of their time. But History sometimes appears suddenly and occupies a place unexpected. We try to see how, when and where.
\end{abstract}

Keywords: literature, world war, history, autobiography, television

\section{INTRODUCTION}

L'Histoire est-elle toujours un passage obligé pour les écrivains belges d'expression française ? C'est à cette question que nous chercherons ici à répondre en nous penchant paradoxalement sur des romanciers qui, tout en dépeignant très précisément leur époque, veillent en général à éviter la grande Histoire. C'est en effet l'histoire avec une minuscule qui intéresse les romanciers étudiés ci-dessous : ils s'attachent tous les quatre à dépeindre, avec des moyens très différents les uns des autres, de façon plus ou moins réaliste, plus ou moins intimiste, plus ou moins poétique, le monde quotidien des hommes, des femmes et des enfants de leur temps. 
Mais l'Histoire, de manière tout à fait inattendue, à la façon du retour du refoulé théorisé jadis par Sigmund Freud, se glisse parfois entre les lignes et occupe subrepticement une place qui lui était jusque-là refusée.

Hormis ce rapport partagé à l'Histoire événementielle et à l'histoire quotidienne, les quatre écrivains belges francophones envisagés dans ce parcours ne présentent entre eux que peu de points communs - si ce n'est qu'ils me sont tous chers, à différents égards, et que j'ai quelque peu étudié leur œuvre respective. Il s'agit, par ordre chronologique, d'André Baillon (1875-1932), de Georges Simenon (19031989), d'Eugène Savitzkaya (né en 1955) et de Jean-Philippe Toussaint (né en 1957). Ils vont être commentés ici les uns après les autres plus ou moins longuement.

\section{ANDRÉ BAILLON : LA CANNELLE AU LIEU DU CANON}

Avec Verhaeren, Maeterlinck et quelques autres grands noms de la littérature belge, André Baillon appartient à la famille, aujourd'hui presque disparue, des écrivains flamands francophones : il est né en Flandres, mais, ayant vécu, une fois adulte, principalement à Bruxelles puis à Paris, il a écrit en français toute son œuvre. Celle-ci a connu une gestation lente et douloureuse : influencé à ses débuts par Flaubert et Baudelaire ${ }^{1}$, l'écrivain se mêle à la vie littéraire bruxelloise ${ }^{2}$ et publie dans la revue Le Thyrse, entre 1899 et 1903, de « la prose lyrique, avec un penchant pour le morbide, $[\ldots]$ et quelques nouvelles naturalistes à la manière de Maupassant qui tracent un portrait satirique de la petite bourgeoisie : deux extrêmes - on croirait lire deux auteurs différents - que Baillon ne concilie pas encore $»^{3}$. Puis il connaît une très longue crise d'inspiration : il continue à écrire mais ne parvient pas à être satisfait de ses projets et n'achève aucun de ceux-ci.

Après plus de dix ans d'errance et de silence éditorial, deux circonstances lui permettent de sortir de l'impasse et de concevoir une écriture qui lui soit propre et qui convienne à son propos. Il s'agit d'abord d'une rencontre amoureuse : en 1912, Baillon, alors marié à Marie, une femme sensible, bonne, maternelle mais peu favorisée culturellement, s'éprend d'une musicienne nommée Germaine Lievens, femme intelligente et cultivée auprès de laquelle il s'épanouit artistiquement ${ }^{4}$. Il s'agit ensuite

\footnotetext{
${ }^{1}$ Voir à ce sujet, R. Trousson, « Postface au Pénitent exaspéré», in A. Baillon, La Dupe, Le Pénitent exaspéré, Bruxelles, Labor, coll. « Archives du futur », 1988, p. 177-209.

${ }^{2}$ Voir à ce sujet P. Aron, «Les illusions perdues d'André Baillon », in B.-O. Dozo, A. Glinoer (dir.), Textyles, $\mathrm{n}^{\circ}$ 46, Figurations de la vie littéraire, 2015, p. 59-73.

${ }^{3}$ F. Denissen, André Baillon. Le gigolo d'Irma Idéal, Bruxelles, Labor, coll. «Archives du futur », 2001, p. 98.

${ }^{4}$ Voir A. Baillon, G. Lievens, « Correspondance mars 1912 - juin 1913. Ou le début laborieux d'un grand amour », établie et éditée par Frans Denissen, dans Les Nouveaux Cahiers André Baillon, $\mathrm{n}^{\circ}$ 10-11, 2012-2014, p. 9-73. Voir aussi l'analyse de cette correspondance: S. Lemoine, Entre folie et lucidité,
} 
d'une circonstance proprement historique : la Première Guerre mondiale. Baillon, qui a 39 ans quand éclate le conflit, ne doit pas prendre les armes. Bien plus, il est encouragé à l'inaction: il travaillait pour un quotidien et, une fois la Belgique envahie par les troupes allemandes, le gouvernement belge en exil décide de verser une pension aux journalistes pour qu'ils ne soient pas contraints de collaborer avec l'occupant. Baillon, devenu rentier, a alors tout le loisir d'écrire et il rattrape le temps perdu en se montrant soudain très productif. C'est en effet durant l'Occupation, à partir de 1915, qu'il rédige ses premiers romans : Histoire d'une Marie, Moi quelque part qui deviendra En sabots, Par fil spécial et, semble-t-il, Délires, son chef-d'œuvre à mes yeux ${ }^{5}$.

La guerre a donc contribué à l'édification de cette œuvre originale. Pourtant, on serait bien en peine d'en trouver la moindre trace dans les romans cités ci-dessus. C'est que Baillon y traite des thèmes qui le poursuivent depuis toujours et qu'il ne parvenait pas à exploiter auparavant. De quoi s'agit-il ?

Son œuvre est riche et complexe, mais il est sans doute légitime de la caractériser par deux oppositions : d'une part, elle oscille entre autobiographie au sens strict et biographie plus ou moins imaginaire d'êtres proches (notamment de Marie, sa première femme), d'autre part, elle se divise entre romans consacrés à des thématiques liées à la vie quotidienne du peuple (des paysans, des petits journalistes ou des prostituées) et romans centrés sur le thème de la folie (Baillon ayant été interné à l'hôpital psychiatrique de la Salpêtrière à deux reprises, en 1923 et en 1924). Certains romans échappent cependant à cette seconde opposition : ceux dans lesquels, à la fin de sa vie, il se penche sur le récit de sa propre enfance (de l'avis presque général, ces romans sont considérés comme mineurs dans l'œuvre ${ }^{6}$ ).

Cette double opposition nous permet de proposer le tableau synthétique suivant :

\begin{tabular}{|l|l|l|}
\hline & \multicolumn{1}{|c|}{ Romans autobiographiques } & $\begin{array}{l}\text { Romans biographiques consacrés à des } \\
\text { proches }\end{array}$ \\
\hline Romans du peuple & $\begin{array}{l}\text { Seconde partie d'Histoire d'une Marie } \\
(1921), \text { En sabots (1922), Par fil } \\
\text { spécial (1924) }\end{array}$ & $\begin{array}{l}\text { Première partie d'Histoire d'une Marie } \\
\text { (1921), Zonzon Pépette (1923) }\end{array}$ \\
\hline Romans de la folie & $\begin{array}{l}\text { Délires (1927), Un homme si simple } \\
(1925), \text { Chalet 1 (1926) }\end{array}$ & Le Perce-oreille du Luxembourg (1928) \\
\hline Romans de l'enfance & $\begin{array}{l}\text { Le Neveu de Mademoiselle Autorité } \\
(1930), \text { Roseau (1931) }\end{array}$ & \\
\hline
\end{tabular}

une analyse de l'ethos baillonnien. Étude de l'énonciation au sein de la correspondance amoureuse d'André Baillon, mémoire de master, Université de Liège, 2015, p. 28-46.

${ }^{5}$ Sur cette chronologie, voir F. Denissen, André Baillon. Le gigolo d'Irma Idéal, op. cit., p. 204.

${ }^{6}$ Au sujet de ces romans, voir M. Ch. Gnocchi, «Je, neveu. Relecture d'une œuvre dite "mineure" d'André Baillon », in P. Aron, L. Brogniez (dir.), Textyles, $\mathrm{n}^{\circ}$ 47, Bruxelles, une géographie littéraire, 2015. 
Il y aurait beaucoup à dire à propos de ce schéma, forcément réducteur. L'appellation " roman du peuple » est évidemment discutable, surtout en ce qui concerne la part autobiographique de l'œuvre, qui met d'abord et avant tout en scène un frère jumeau de l'auteur, c'est-à-dire un artiste doublé d'un bourgeois déclassé. Toujours est-il que Baillon, souvent comparé à Charles-Louis Philippe, a vu ses livres classés dans la catégorie « littérature populiste », notamment parce qu'il était publié par un éditeur, Rieder, spécialisé dans ce domaine particulier. Mais, en poursuivant ces considérations, nous risquons de nous écarter trop loin de notre sujet ${ }^{7}$. Il importe, en revanche, de souligner que, quelle que soit la case envisagée, l'Histoire événementielle n'a pas ici droit de cité, pas même cette Première Guerre mondiale qui a pourtant rendu possible la rédaction de l'œuvre. La guerre ne joue de rôle direct ni dans l'autobiographie de l'écrivain, qui se consacre surtout à des événements antérieurs ni, pour la même raison, dans ses biographies de proches.

Il en va de même pour la seconde opposition de notre tableau : la folie et le peuple. Si Michel Foucault nous a prouvé que la folie avait une histoire, celle-ci a trait à ses représentations et à ses traitements bien davantage qu'à sa nature. Les asiles décrits par Baillon dans Un homme si simple ou Chalet 1 sont datés historiquement, mais là n'est pas l'essentiel du propos de l'écrivain, qui se penche sur des désordres intimes. Et le délire, dans sa splendeur comme dans sa misère, présente des aspects intemporels. Quant au peuple, il est décrit dans une sorte de présent immuable, qu'il s'agisse des paysans du roman par fragments En sabots ou des prostituées d'Histoire d'une Marie ou de Zonzon Pépette, fille de Londres, qui exercent ce qu'en français, selon une périphrase magnifique d'hypocrisie, on appelle «le plus vieux métier du monde ». La situation est plus complexe en ce qui concerne les journalistes, croqués avec ironie dans Par fil spécial: leur profession est des plus modernes, tout à fait en phase avec l'air du temps et avec l'évolution technique Baillon décrit d'ailleurs le rôle des machines dans une rédaction. Mais, le romancier, qui a travaillé pour le quotidien belge La Dernière Heure de 1909 à 1920, n'évoque pas la presse de guerre, puisqu'il est demeuré sans emploi durant celle-ci.

D'après Frans Denissen, son biographe, certains critiques reprochèrent à Baillon son silence au sujet du conflit, qui trahissait à leurs yeux un « manque total de "patriotisme" 》 et un "égocentrisme » l'empêchant "de s'intéresser au monde extérieur $»^{8}$. Il ne nous semble pas que ces deux arguments, dictés par l'idéologie haineuse du temps, doivent nous retenir ici. Une autre piste de réflexion, plus intéressante, se propose à nous à travers les propos de l'écrivain. En effet, comme il l'explique en comparant, après la sortie de Par fil spécial, son ancien métier de journaliste et celui d'écrivain, Baillon a besoin de temps pour écrire un roman :

\footnotetext{
${ }^{7}$ Renvoyons plutôt à l'ouvrage de M. Ch. Gnocchi, Le Parti pris des périphéries. Les « Prosateurs français contemporains » des éditions Rieder (1921-1939), Bruxelles, Le Cri/Ciel-ULB-Ulg, 2007.

${ }^{8}$ F. Denissen, André Baillon. Le gigolo d'Irma Idéal, op. cit., p. 202.
} 
Un reporter est obligé de voir et d'écrire vite. Pour voir, je dois ignorer que je regarde. Quant à écrire, je prends mon temps. Lorsque je vivais ma vie d'En sabots, je ne pensais pas qu'il en sortirait un livre. Il m'a fallu des années : c'est une somme de souvenirs et de nostalgie ${ }^{9}$.

Cette dernière citation nous permet d'avancer dans notre propos. Faisons le point: Baillon, qu'il parle de lui ou de ses proches, dépeint de toute façon son époque, mais il le fait en romancier et non en journaliste. Quelle est la différence ? Si l'on s'appuie sur le propos de Baillon, le journaliste voit et écrit immédiatement, dans l'urgence, tandis que le romancier regarde, écrit lentement et dans un temps second, sous le coup de la nostalgie, une fois les faits intégrés. De ce point de vue, Baillon aurait peut-être écrit un livre sur la guerre s'il avait vécu plus longtemps, mais il a mis fin à ses jours en 1932 alors qu'il allait avoir 57 ans.

Peut-être : rien n'est moins sûr. La nature des faits du passé est aussi à prendre en considération, bien entendu. Baillon, on l'aura compris, n'est pas un peintre de bataille. Quand il ne monte pas sur les grands chevaux de la folie, il voue sa plume à la description de la vie quotidienne. Le bref art poétique qu'il a fait paraître dans Le Thyrse en 1921 est clair à cet égard: il s'y oppose aux écrivains qui ne s'intéressent « qu'aux héros d'exception : géniaux, divins, gonflés de sentiments sublimes », préférant pour sa part « si humble soit-il, comprendre que chaque être est précisément à l'exception des autres et saisir la nuance $»{ }^{10}$.

Or, l'existence des êtres uniques et ordinaires peut changer de nature en fonction des grands événements historiques. C'est ici que la Première Guerre mondiale fait tout de même une brève apparition dans l'œuvre de Baillon, au gré d'un court texte intitulé « En pays occupé », dernière nouvelle du recueil La vie est quotidienne, qui est paru en 1929 chez Rieder et qui n'a jamais été réédité comme tel depuis lors ${ }^{11}$. Dans cette nouvelle, c'est à travers son implication sur la vie de tous les jours que la guerre et l'occupation jouent leur partition : il y est surtout question du rationnement alimentaire. Le beau rôle y est donné à celle que le narrateur appelle « Ma Nounouche », c'est-à-dire à Germaine Lievens, qui a acheté au début du conflit deux kilos de cannelle. Le narrateur se fâche de cet achat inconsidéré en temps de disette : «Voyons, c'est idiot. Avec l'argent de la cannelle, tu aurais pu acheter d'autres haricots. On achète cent grammes de cannelle ; cent cinquante grammes de cannelle. Mais deux kilos ! Nous en avons pour cent ans ! ${ }^{12}$ Puis, une fois sa colère calmée,

\footnotetext{
${ }^{9}$ Réponse de Baillon au critique littéraire Frédéric Lefèvre qui l'interroge dans Les Nouvelles littéraires au moment de la parution de Par fil spécial (1924) citée par M. Grodent, dans «Lecture» d'A. Baillon, Par fil spécial, Bruxelles, Labor, coll. « Espace nord », 1995, p. 209.

${ }^{10}$ A. Baillon, «Traité de littérature » [1921], Textyles, n 6, André Baillon le précurseur, 1989, p. 143.

${ }^{11}$ Dans A. Baillon, Le Chien-chien à sa mémère, Le Bouscat, Finitude, 2013, sont réédités 11 des 14 récits de La vie est quotidienne, ainsi que deux textes de Baillon ayant paru dans L'Humanité.

${ }^{12}$ A. Baillon, La vie est quotidienne, Paris, Rieder, 1929, p. 211.
} 
il fait de cette réserve inhabituelle un sujet de plaisanterie récurrente. Les mois, les années passant, l'occupation s'éternisant et la qualité de la nourriture diminuant, cette cannelle devient une denrée précieuse : « [Ma Nounouche] était trop modeste. Elle se montrait fière quand même. Et je ne riais plus. Les amis qui venaient ne riaient pas non plus. Eux qui devaient avaler ces tartes, ces crêpes - et sans cannelle ${ }^{13}$.

La cannelle et les petits conflits entre les époux plutôt que le canon et le grand conflit entre les peuples: telle est la vision de l'Histoire qui transparait en douce dans l'œuvre originale, ironique et touchante d'André Baillon.

\section{GEORGES SIMENON ET LES «CONVULSIONS DE L’ÉPOQUE »}

Nous nous attarderons beaucoup moins longtemps sur le second écrivain belge francophone envisagé ici: Georges Simenon. Et cela, pour plusieurs raisons. D'abord, le père du commissaire Maigret est, c'est peu de le dire, beaucoup plus célèbre qu'André Baillon et je ne vais pas faire l'injure à mon lecteur de le lui présenter. Ensuite, la question de son rapport à l'Histoire a déjà été étudiée, notamment par Jacques Dubois et Benoît Denis dans leur irremplaçable introduction au premier tome qui lui est consacré dans la prestigieuse collection de La Pléiade et par Pierre Assouline dans sa biographie du romancier ${ }^{14}$. Nous nous contenterons donc de rappeler certains faits dans la mesure où, étrangement, ils résonnent avec les constatations faites ici au sujet d'André Baillon. "Étrangement», dis-je, car les deux auteurs paraissent aux antipodes l'un de l'autre. Leur lectorat, leur place dans le champ littéraire, leur postérité diffèrent du tout au tout, ainsi que leur manière de conduire un récit, sérieuse et implacable chez Simenon, ironique et désinvolte chez Baillon. Tous deux sont en quête d'une forme de simplicité dans l'écriture, mais il s'agit de la quête d'une inaccessible étoile, pure et lointaine, pour l'auteur d'Un homme si simple, tandis que le père de Maigret atteint à cet égard son but sans coup férir, rien ne semblant plus simple pour lui que d'être simple, alors que rien ne paraît plus compliqué pour son aîné. Les deux romanciers ont pourtant en commun de se pencher sur l'homme ordinaire («l'homme nu », dira Simenon) et non sur les « héros » et, à ce titre, tous deux ont d'ailleurs été un temps considérés comme des écrivains populistes ${ }^{15}$.

Toujours est-il que, comme Baillon, Simenon a besoin de temps pour digérer un événement avant de l'inclure dans la fiction. Et, lui aussi, il se consacre non à des collectivités, mais à des trajectoires individuelles et contemporaines. Le plus sou-

${ }^{13}$ Ibidem, p. 214.

${ }^{14}$ Voir P. Assouline, Simenon, Paris, Gallimard, coll. «Folio », 1996 [1992], p. 403-408.

${ }^{15}$ Nous avons déjà rapidement souligné ce point en ce qui concerne Baillon. Quant à Simenon, voir B. Denis, J. Dubois, "Introduction», dans G. Simenon, Romans, tome I, Paris, Gallimard, coll. «Bibliothèque de La Pléiade», 2003, p. XXVIII-XXXII. [Une note précise que ce passage de cette Introduction à quatre mains est dû à Jacques Dubois.] 
vent, ce qui le singularise, ces trajectoires sont centrées sur une crise existentielle. L'Histoire s'en trouve quelque peu exilée, mais pas complètement. Citons à ce sujet Jacques Dubois :

Cependant, pas plus que Simenon n'a échappé au champ littéraire, son œuvre n'a pu entièrement se soustraire aux grandes convulsions de l'époque. La liste est respectable des romans qui touchent à de grandes questions du temps ou qui en évoquent les acteurs : le colonialisme dans Le Coup de lune (1933), le régime soviétique dans Les Gens d'en face (1933), les attentats anarchistes dans Le Suspect (1938), une occupation de type de fasciste ou communiste dans La neige était sale (1948). À chaque fois néanmoins, ces grands faits du temps ne sont pas décrits comme tels mais représentés allusivement, à travers une aventure toute personnelle ou sous une forme décontextualisée. [...] Pedigree (1948) dit une grève ouvrière importante depuis l'effroi qu'éprouve Élise Mamelin face à l'événement, et Le Train (1961) transforme l'évacuation de 1940 en liaison amoureuse. Mais la littérature ne parle-t-elle pas aussi bien de l'Histoire lorsqu'elle emprunte des voies obliques que quand elle peint de grandes fresques ${ }^{16}$ ?

On ne peut mieux dire!

Le tableau brossé ci-dessus pourrait être complété par d'autres titres, comme Le Clan des Ostendais (1947), qui met en scène un marin flamand coincé en France au début de la guerre. Mais, même si nous obtenons ainsi, selon l'expression de Dubois, une liste respectable, celle-ci demeure courte si l'on songe à l'immense production de Simenon, qui a publié près de deux cents romans sous son nom et presque autant sous divers pseudonymes! Dans l'ensemble, l'Histoire se fait donc discrète : sa place est à la fois conditionnée par des destins individuels et somme toute assez rare. Il s'agit là aussi de retours subreptices.

\section{EUGÈNE SAVITZKAYA : \\ L'HISTOIRE D'AVANT DANS L'APRÈS-COUP}

Le troisième écrivain envisagé ici présente un cas de figure très différent: il s'agit d'Eugène Savitzkaya, un auteur qui, pour avoir derrière lui déjà une quinzaine de recueils poétiques, une vingtaine de romans, un volume de correspondances (avec Hervé Guibert), une dizaine de pièces de théâtre et une bonne vingtaine d'ouvrages réalisés avec divers plasticiens, est toujours en pleine activité créatrice - ses deux derniers livres, Fraudeur et $\grave{A}$ la cyprine, sont parus en 2015 aux éditions de Minuit. Alors que Baillon avait subi la première occupation de la Belgique par les Allemands, que Simenon se souvenait de la fin de la Première Guerre mondiale et avait vécu durant la Seconde à l'âge adulte, Savitzkaya, qui est né en 1955, n'a

\footnotetext{
${ }^{16}$ Ibidem, p. LVII. [La même note nous indique que ce passage de l'Introduction est également de Dubois.]
} 
connu ni conflits armés ni invasions. Certes, l'Histoire a poursuivi son implacable chemin sur terre, contrairement à ce qu'a pu penser Francis Fukuyama aux débuts des années 1990, mais elle a laissé la Belgique relativement en paix depuis 1945 ou du moins depuis la "Question royale », qui a agité le pays en 1950-1951. Pour un citoyen belge de la génération de Savitzkaya ou des générations postérieures, la grande Histoire événementielle présente toujours des traits exotiques, qu'il s'agisse d'exotisme spatial ${ }^{17}$ ou d'un exotisme temporel. L'Histoire s'écrit donc alors au second degré.

En outre, contrairement aux deux auteurs précédents, qui sont avant tout des réalistes, Savitzkaya est autant un poète qu'un romancier et la littérature qu'il produit, si elle a trait au réel, se montre volontiers fantasmatique. Son sujet principal est l'enfance, qu'il traite de multiples manières. Certains de ses romans donnent directement la parole à un enfant, de façon homodiégétique et autodiégétique [par exemple Mentir (1977) ou La Disparition de maman (1982)], d'autres s'appuient sur un narrateur extérieur (donc hétérodiégétique), mais procèdent à une focalisation interne sur un enfant [Les morts sentent bon (1984) ou Fraudeur (2015)]. Or, si certains petits garçons et certaines petites filles, de par le monde, sont ballotés par l'Histoire, l'enfance est sans doute la période de la vie qui se soucie le moins directement de celle-ci. Dans d'autres romans encore, Savitzkaya donne, cette fois, la parole à un adulte, mais celui-ci observe alors son fils ou sa fille dans le cadre de l'intimité familiale [Marin mon cœur (1992), Exquise Louise (2003)]. Ces derniers titres présentent certainement un monde moins intemporel et un peu moins fantasmatique que ceux centrés directement sur une conscience enfantine : il s'agit assurément d'un père contemporain, donc inscrit historiquement dans son époque, même s'il demeure très singulier $^{18}$. Mais nul événement collectif n'y vient déranger la contemplation paternelle.

Au sein de la grande production de Savitzkaya, un roman fait pourtant figure d'exception: Fou civil (1999), qui s'apparente à une autofiction - l'écrivain y dépeint son quotidien à Liège et explique ses difficultés financières. Alors que, jusque-là, la seule figure historique apparue dans l'œuvre d'Eugène Savitzkaya était Elvis Presley, personnage principal d'un roman au titre ironique Un jeune homme trop gros (1978) (mais il s'agissait d'un Elvis imaginaire, rêvé à partir de photographies ${ }^{19}$ ), c'est de manière très détournée que survient vingt-deux ans plus tard un individu beaucoup moins sympathique :

${ }^{17}$ Après 1950, l'événement national le plus important a eu paradoxalement lieu en Afrique centrale, quand, en 1960, le Congo a quitté son statut de colonie belge pour accéder à l'indépendance.

${ }^{18}$ Je ne peux pas ici ne pas renvoyer à un article que j'ai co-écrit avec feu mon père: Christian, Laurent Demoulin, «L'enfant dieu selon Savitzkaya », dans La Clinique lacanienne, $\mathrm{n}^{\circ} 10$, Les nouveaux rapports à l'enfant, Érès, 2006, p. 35-51, disponible en ligne : http://www.cairn.info/revue-la-clini que-lacanienne-2006-1-p-35.htm.

${ }^{19}$ Voir à ce sujet D. Bajomée, « Les chambres noires d'Elvis. Pour une lecture d'Un jeune homme

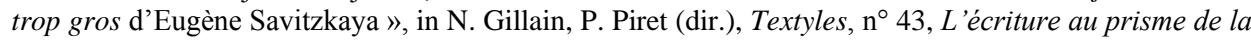
photographie, Bruxelles, Le Cri, 2013, p. 97-107. 
Il dort entre des draps d'une finesse extrême, usés par les hanches et les épaules d'une grand-mère russe qui repose en terre brabançonne. En rêve, il a croisé le regard du Docteur Mengele et il ne peut l'oublier. Il est, selon son penchant naturel, doux et paisible, mais il ne rechigne pas aux calembredaines et il aime en découdre si cela s'avère nécessaire ${ }^{20}$.

C'est donc, en plein milieu d'un autoportrait à la troisième personne, et par le biais d'un cauchemar, que l'Histoire, dans sa version la plus monstrueuse, fait une très courte apparition, sous le nom du sinistre médecin d'Auschwitz, comme pour nous indiquer que nul ne peut y échapper.

Mais cet extrait est intéressant aussi dans la mesure où l'auteur y évoque, à travers le personnage de la grand-mère, ses origines russes. Car si Savitzkaya n'a pas eu à subir les soubresauts de l'Histoire, ses parents les ont connus, notamment à travers l'exil et la guerre, son père ayant atterri en Belgique en venant de Pologne et sa mère de Russie. Jeune homme, Eugène Savitzkaya, peut-être trop occupé par le caractère fantasmatique de ses écrits, ne voyaient pas là de sujets qui méritaient d'être exploités, comme il s'en est expliqué en 1982 à son ami, le regretté Hervé Guibert, qui semblait s'en étonner :

E. S. : Je n'ai pas de famille non plus, ou elle est sans intérêt. Mes parents ont tous deux quitté leur pays natal, au moment de la guerre, réquisitionnés comme travailleurs en Allemagne. Mon père était orphelin, il travaillait dans une petite ferme en Pologne, pas loin de Cracovie. Puis d'Allemagne, il est venu dans les charbonnages de Liège. Ma mère est née en 1926, en Russie blanche, près de Smolensk, son grand-père était un petit propriétaire terrien. Ses parents ont déménagé en Ukraine et c'est d'Ukraine qu'elle est partie en Allemagne pendant la guerre, puis de là en Belgique. Elle a quitté sa famille, et elle ne m'a jamais expliqué pourquoi elle n'a pas cherché à la revoir. C'est un mystère.

H. G. : Ce n'est pas une matière qui t'intéressait?

E. S. : J'ai voulu à plusieurs reprises poser des questions, mais les réponses qu'elle me donnait ne me disaient rien. La tentative a avorté. Je préfère m'introduire clandestinement dans les familles des autres ${ }^{21}$.

Avec le temps, Savitzkaya semble changer doucement d'avis à ce sujet. Il n'a certes pas entrepris de grand récit familial, parce qu'il reste fidèle à une vision essentiellement poétique de la littérature et que la narration n'est pas pour lui primordiale, mais l'histoire de ses parents, petit à petit, se met à occuper de plus en plus de place dans ses écrits. Dans Fou trop poli (2004), la Russie et la Pologne des origines sont évoquées, mais leur apport éventuel est vite dénié :

${ }^{20}$ E. Savitzkaya, Fou civil, Paris, Flohic, 1999, p. 11.

${ }^{21}$ Guibert (Hervé) «Une rencontre avec Eugène Savitzkaya », Minuit, n ${ }^{\circ} 49$, mai 1982, p. 6. Une note, dans la correspondance échangée entre les deux écrivains, nous apprend toutefois qu'ils avaient projeté de réaliser ensemble un voyage à Starabouda, sur les traces de la mère de l'écrivain belge (H. Guibert, E. Savitzkaya, Lettres à Eugène. Correspondance 1977-1987, Paris, Gallimard, p. 127). 
Ceux qui disent que la Russie est blafarde et grise se trompent lourdement de couleur, car elle n'est pas grise, mais bleue. [...]

J'ai tout désappris très vite en ce qui concerne ces pays-là. J'ai désappris tant, que je sème, sans crainte des malédictions, mes cheveux chez la coiffeuse, mes dents chez le dentiste et d'autres productions de mon corps russo-polonais chez les médecins ${ }^{22}$.

Le corps est donc ici le seul dépositaire de l'Histoire, et c'est un corps dont on se débarrasse.

Il en va tout autrement dans deux textes qui forment un couple : Célébration d'un mariage improbable et illimité (2002) et Nouba (2007), deux variantes de la même pièce de théâtre (improbable elle aussi). Les invités conviés à un mariage y évoquent, entre autres sujets, ce que l'époux apporte à l'épouse et vice versa. Dans la seconde version, on lit ce passage :

[...] l'homme est polonais, la femme est russe, la femme invoque le domovoï et l'homme crie, que Pieroun te pourfende et le tonnerre gronde et la foudre tombe et le lait tourne et la soupe surit, l'homme est russe, la femme est polonaise $[\ldots]^{23}$.

Cette réplique, qui rend réversibles les deux origines slaves de l'auteur, donne à penser qu'une de ses sources d'inspiration de cet texte est sans doute l'union de ses parents $^{24}$.

Enfin, examinons rapidement le dernier roman paru, Fraudeur (2015), qui se présente comme une réécriture du premier, Mentir (1977). Le récit se centre à nouveau sur l'enfance de l'auteur et, cette fois, aborde la question historique des origines. Mais ce thème ne surgit ici que par éclats, de façon brève et brûlante, au milieu de la peinture du quotidien de l'enfant découvrant le monde autour de lui. Le lecteur apprend ainsi, au détour d'une page, que le père a été « mineur dans la région de Liège après avoir boxé dans les baraquements en Allemagne pour distraire les troupes américaines $»^{25}$. Plus haut, lorsque le narrateur décrit la mère qui « parle aux peupliers $\gg{ }^{26}$, survient une bribe de souvenir :

Jeune, elle aimait chanter. Aux Jeunesses communistes, elle imitait les aviateurs, ses longs cheveux cachés dans une casquette. Quel Allemand la séduisit ? Vint un enfant en pleine guerre ${ }^{27}$.

Et le chapitre de s'interrompre, laissant le lecteur sur une question sans réponse, suivie d'une affirmation lapidaire qu'aucun développement ultérieur ne viendra

${ }^{22}$ E. Savitzkaya, Fou trop poli, Paris, Éditions de Minuit, 2005, p. 13-14.

${ }^{23}$ E. Savitzkaya, Nouba, Liège, Yellow Now, 2007, p. 64.

${ }^{24}$ Cette fois, nulle excuse filiale à évoquer : c'est sans vergogne que je renvoie à mon article sur le sujet : L. Demoulin, «Le dispositif Célébration/Nouba », dans Textyles, ${ }^{\circ} 44$, Eugène Savitzkaya : le corps des mots, Bruxelles, Samsa, 2011.

${ }^{25}$ E. Savitzkaya, Fraudeur, Paris, Éditions de Minuit, 2015, p. 121.

${ }^{26}$ Ibidem, p. 74.

${ }^{27}$ Ibidem. 
déployer. Nous sommes bien dans le mystère, comme Savitzkaya le déclarait à Guibert en 1982.

La grande Histoire, chez Eugène Savitzkaya, gagne donc sans cesse du terrain, à travers le retour de la petite histoire familiale, mais de façon désordonnée, subite et brève.

\section{JEAN-PHILIPPE TOUSSAINT : FLASH D’HISTOIRE À LA TÉLÉVISION}

Le dernier écrivain commenté ici, Jean-Philippe Toussaint, est né en 1957 : il appartient à la même génération que Savitzkaya et n'a donc pas non plus eu à survivre à une guerre. Si son grand-père paternel, Juozas Lanskoronskis, était Lituanien, ses parents n'ont pas connu les mêmes affres que ceux de Savitzkaya.

Et, bien que ses romans, centrés sur quelques personnages, fassent voyager les lecteurs dans l'espace (notamment en France, en Allemagne, en Chine et au Japon), ils ne leur proposent pas de voyage dans le temps. Résolument, Toussaint est un écrivain contemporain, qui cherche à décrire le monde qui l'entoure aujourd'hui.

Se pose alors la question de savoir si le présent fait partie de l'Histoire. Immanquablement, pourrait-on répondre, il finit par y entrer dès qu'il dure un petit peu. Comme le premier roman de Toussaint, La Salle de bain, est paru en 1985, l'Histoire a peut-être rattrapé son œuvre : il est ainsi question dans Faire l'amour (2002) d'une technologie qui est déjà en train de disparaître : le fax. Le roman s'en trouve irrémédiablement daté !

Quoi qu'il en soit, même si c'est dans son évolution que Toussaint cherche à happer le présent, il échappe, comme Baillon, à la grande Histoire dans la mesure où il dépeint la vie courante et non les événements collectifs fédérateurs ${ }^{28}$. Les éléments et les objets d'ordinaire négligés par la littérature et pourtant si présents dans nos existences, tels que la télévision, les appareils-photos, les grands magasins, les téléphones portables, les cartes magnétiques, les villes nouvelles, les avions, les équipes médicales des urgences ou le permis de conduire jouent un grand rôle dans ses récits magnifiques et inattendus, ironiques ou poétiques.

Sauf erreur de ma part, une seule page, dans un seul de ses neuf romans, laisse, une fois encore, la grande Histoire se glisser entre les mots. Il s'agit d'un long et beau passage de La Télévision (1997) : le narrateur, qui vit à Berlin, y décrit une séance de zapping abrutissant et laisse les images déferler :

Partout c'était les même images indifférentes, sans marges et sans en-têtes, sans explication, brutes, incompréhensibles, bruyantes et colorées, laides, tristes, agressives et joviales,

\footnotetext{
${ }^{28}$ Avec une exception pour la coupe du monde de football de 2006, dont la finale est racontée dans La Mélancolie de Zidane (2006).
} 
syncopées, équivalentes, c'étaient des séries américaines stéréotypées, c'était des clips, c'était des chansons en anglais, c'était des jeux télévisés, c'était des documentaires, c'était des scènes de film sorties de leur contexte, des extraits, c'était des extraits, c'était de la chansonnette, c'était vivant, le public battait des mains en rythme, c'était des hommes politiques autour d'une table, c'était un débat, c'était du cirque, c'était des acrobaties, c'était un jeu télévisé, c'était le bonheur, des rires de stupéfaction incrédule, des embrassades et des larmes, c'était le gain d'une voiture en direct, des lèvres qui tremblaient d'émotion, c'était des documentaires, c'était la deuxième guerre mondiale, c'était une marche funèbre, c'était des colonnes de prisonniers allemands qui marchaient lentement sur le bord de la route, c'était la libération des camps de la mort, c'était des tas d'ossements sur la terre, c'était dans toutes les langues, [...] c'était des informations, c'était des inondations, c'était du football, c'était des jeux télévisés, c'était un animateur avec des fiches $[\ldots]^{29}$.

L'énumération continue encore pendant plusieurs pages, alternant les images graves et futiles, dans une sorte de mise à plat dénonçant le tout-se-vaut du propos télévisuel. L'Histoire est présente, fugitive et tragique, avec à nouveau le motif de la Seconde Guerre mondiale et celui des camps de la mort, que résume le nom de Mengele chez Savitzkaya. Chez ces deux romanciers si dissemblables, une forme de retour du refoulé historique passe par la même évocation.

\section{CONCLUSIONS}

Nous avons présenté ici, plus ou moins longuement, quatre écrivains belges francophones qui ne se voient jamais ainsi associés par la critique. Ils ont en commun de préférer l'intime au collectif et de ne chercher ni à produire des tableaux historiques, ni à relater de grands événements et ils ne s'attachent pas à des héros mais à des hommes ordinaires (ou à des enfants dans le cas de Savitzkaya).

Et pourtant, aucun des quatre ne parvient à se soustraire tout à fait à l'Histoire. Celle-ci fait retour essentiellement de trois façons :

Indirectement, presque contre le gré des auteurs, par le biais de la description du présent : les paysans des années 1920 décrits par Baillon ont l'air d'échapper au temps, mais ils ont disparu aujourd'hui ; les rapports familiaux qui structurent la plupart des romans «durs » de Simenon datent assurément d'avant mai 1968 ; le père narrateur que postule Marin mon cœur de Savitzkaya aurait été impensable au début du $\mathrm{XX}^{\mathrm{e}}$ siècle et Toussaint commente, lors d'un entretien, sa description d'un Boeing en précisant avec humour que Proust, sur le sujet, s'est montré peu disert...

Par le biais de l'histoire familiale, timidement, mais avec insistance, chez Savitzkaya.

\footnotetext{
${ }^{29}$ J.-P. Toussaint, La Télévision, Paris, Minuit, 1997, p. 22-23.
} 
Subrepticement, et non plus dans l'immédiat mais après que plusieurs années ont passé, de manière consciente cette fois, de biais, par fulgurations ou entre parenthèses : par des évocations de la grande Histoire, plus précisément de la guerre ou des camps, à travers un cauchemar (Savitzkaya), un état d'abrutissement dû à une overdose d'images cathodiques (Toussaint), la sensibilité d'un personnage médiocre (Simenon) ou la poudre, inoffensive et parfumée, de la cannelle (Baillon). 


\section{BIBLIOGRAPHIE}

Aron, P. (2015). «Les illusions perdues d'André Baillon », in B.-O. Dozo, A. Glinoer, Textyles, 46, Figurations de la vie littéraire.

Assouline, P. (1996). Simenon, Paris : Gallimard, coll. « Folio».

Baillon, A. (1929). La vie est quotidienne, Paris : Rieder, coll. « Les prosateurs contemporains ».

- (1989). « Traité de littérature » [1921], Textyles, 6, André Baillon le précurseur, 143.

- (2013). Le Chien-chien à sa mémère, Le Bouscat : Finitude, 2013.

Baillon, A., Lievens G. (2012-2014). « Correspondance mars 1912 - juin 1913. Ou le début laborieux d'un grand amour », établie et éditée par Frans Denissen, Les Nouveaux Cahiers André Baillon, 10-11, 9-73.

Bajomée, D. (2013). «Les chambres noires d'Elvis. Pour une lecture d'Un jeune homme trop gros d'Eugène Savitzkaya », in N. Gillain, P. Piret, Textyles, 43, L'écriture au prisme de la photographie, Bruxelles : Le Cri, 2013, 97-107

Demoulin, L. (2011). «Le dispositif Célébration/Nouba», Textyles, 44, Eugène Savitzkaya: le corps des mots, Bruxelles : Samsa.

Denis, B., Dubois, J. (2003). «Introduction », in G. Simenon, Romans, tome I, Paris : Gallimard, coll. «Bibliothèque de La Pléiade », xxviii-xxxii.

Denissen, F. (2001). André Baillon. Le gigolo d'Irma Idéal, Bruxelles : Labor, coll. « Archives du futur».

Gnocchi, M. C. (2015). «Je, neveu. Relecture d'une œuvre dite "mineure" d'André Baillon », in P. Aron, L. Brogniez, Textyles, 47, Bruxelles, une géographie littéraire.

Grodent, M. (1995). «Lecture», in A. Baillon, Par fil spécial, Bruxelles : Labor, coll. «Espace nord ».

Guibert, H. (1982). «Une rencontre avec Eugène Savitzkaya », Minuit, 49.

Guibert, H., Savitzkaya, E. (2013). Lettres à Eugène. Correspondance 1977-1987, Paris : Gallimard.

Lemoine, S. (2015). Entre folie et lucidité, une analyse de l'ethos baillonnien. Étude de l'énonciation au sein de la correspondance amoureuse d'André Baillon, mémoire de master, Liège : Université de Liège.

Gnocchi, M. C. (2007). Le Parti pris des périphéries. Les « Prosateurs français contemporains » des éditions Rieder (1921-1939), Bruxelles : Le Cri/Ciel-ULB-Ulg.

Savitzkaya, E. (1999). Fou civil, Paris : Flohic.

- (2005). Fou trop poli, Paris : Minuit.

- (2007). Nouba, Liège : Yellow Now.

- (2015). Fraudeur, Paris : Minuit.

Toussaint, J.-P. (1997). La Télévision, Paris : Minuit.

Trousson, R. (1988). "Postface au Pénitent exaspéré», in A. Baillon, La Dupe, Le Pénitent exaspéré, Bruxelles : Labor, coll. « Archives du futur». 